\title{
Tsafon
}

Revue d'études juives du Nord

$78 \mid 2019$

L'antijudaïsme dans l'Antiquité

\section{Amalec, ou l'ennemi héréditaire dans le midrash rabbinique}

\section{Matthias Morgenstern}

Traducteur : Joseph Kolbl

\section{OpenEdition}

\section{Journals}

Édition électronique

URL : https://journals.openedition.org/tsafon/2402

DOI : 10.4000/tsafon.2402

ISSN : 2609-6420

\section{Éditeur}

Association Jean-Marie Delmaire

\section{Édition imprimée}

Date de publication : 1 décembre 2019

Pagination : 85-108

ISSN : 1149-6630

\section{Référence électronique}

Matthias Morgenstern, «Amalec, ou l'ennemi héréditaire dans le midrash rabbinique », Tsafon [En ligne], 78 | 2019, mis en ligne le 01 décembre 2019, consulté le 28 juin 2022. URL : http:// journals.openedition.org/tsafon/2402 ; DOI : https://doi.org/10.4000/tsafon.2402 


\section{Amalec, ou l'ennemi héréditaire dans le midrash rabbinique}

\section{Matthias Morgenstern*}

Dans la Bible hébraïque, le nom d'Amalec désigne par métonymie le peuple des Amalécites, l'ennemi constant, voire héréditaire d'Israël. Dans la tradition juive et l'ensemble de la littérature rabbinique, ce nom a ainsi une consonance sinistre. La présentation dépréciative se manifeste déjà dans le classement généalogique de l'ancêtre de ce peuple. Dans le livre de la Genèse ${ }^{1}$, Amalec figure comme un petit-fils d'Ésaü (Edom), jumeau et rival du patriarche Jacob, et de ce fait comme un descendant d'Abraham. Ce n'est pas un hasard si cette construction généalogique fait d'emblée d'Amalec, fils d'Éliphaz, un descendant déchu occupant une place à part. Dans Gn 36, 9-14, la structure initiale des douze fils et petits-fils d'Ésaü est encore reconnaissable, au sein de laquelle Amalec se distingue comme le treizième membre ${ }^{2}$. Ce qui frappe par ailleurs, c'est qu'Amalec est le fils d'une concubine (pilegesh) d'Éliphaz, fils aîné d'Ésaü, qui porte le nom de Timna. À l'exception de celle-ci, aucun nom des épouses des fils d'Ésaü n'est cité dans ce passage biblique. Ce verset

\footnotetext{
* Institutum Judaicum, Eberhard Karls-Universität, Tübingen. Article traduit par Joseph Kolbl, Université de Lille, sciences humaines et sociales.

${ }^{1}$ Genèse 36,12 et 16 ; cf. aussi 1 Chroniques 1,36 .

${ }^{2}$ Concernant ce texte, voir T. Hieke, Die Genealogien der Genesis (HBS 39), Fribourgen-Br. 2003, p. 173-191 (notamment p. 186 sq.) et p. 283, ainsi que p. 306 sq. Je tiens à remercier Wolfgang Oswald pour m'avoir indiqué certaines publications et pour les discussions intéressantes que j'ai eues avec lui sur ce texte. En se fondant sur la Genèse (chap. 36), la descendance d'Ésaü, petit-fils d'Abraham, peut être résumée de la façon suivante. Sa première femme et sa troisième lui donnent, chacune, un fils : Éliphaz, fils d'Ada ; Réuel, fils de Basemath. Éliphaz a cinq fils, Reuel en a quatre. Ésaü a en outre trois autres fils avec sa deuxième femme Oholibama, soit douze descendants mâles. Gn 36, 12 ajoute qu'Éliphaz a eu un dernier fils, Amalac, avec sa concubine Timna. Ce qui porte à treize le nombre des descendants d'Ésaü.
} 
12a constitue de toute évidence un ajout postérieur à la source sacerdotale dont la fonction est de dévaloriser d'emblée le «prince (alluf) Amalec » tel qu'il est défini en Gn 36, $16^{3}$.

Les textes midrashiques tiennent compte de la position particulière d'Amalec au sein de la lignée d'Abraham en ajoutant une histoire : selon Berechit Rabba 82, 14 (sur Gn 36, 12), la mention de Timna doit contribuer à la gloire d'Abraham. Les royaumes et les princes non israélites (malkhiyot we-shiltonim) auraient souhaité tellement d'entrer en relation de parenté avec Abraham, que Timna, la sœur de Lotan, prince (alluf) des Horites (Gn 36, 22 et 29), se serait contentée d'être la servante (shifehah) d'Éliphaz. Le Talmud de Babylone (bSan 99b) fait un pas de plus en amplifiant cette narration : selon lui, la princesse (bat melakhim) Timna aurait eu l'intention de devenir prosélyte, c'est-à-dire de se convertir au judaïsme, et s'en serait ouverte à Abraham, Isaac et Jacob. Mais les patriarches, nous dit-on, s'y sont refusés (welo qibelouha). La princesse, sur ces entrefaites, se ravisa et devint l'une des concubines d'Éliphaz en déclarant: «Je préfère être une servante au sein de cette nation plutôt que d'être une maîtresse dans une autre nation $»$.

Ce développement narratif de l'épisode de Timna paraît assez fantaisiste. Dans tous les cas, il vise à chercher, derrière les allusions bibliques, un sens profond des origines et de l'évolution historique du peuple amalécite. Selon le talmudiste, les antécédents remontent à la décision fatidique des patriarches qui n'ont pu se résoudre à autoriser la princesse horite à se convertir au judaïsme ${ }^{5}$. La conséquence en fut sa décision, sans doute par dépit, de se contenter du statut de concubine et ainsi de s'unir ne serait-ce qu'à une lignée secondaire de la famille d'Abraham. Mais sur cette union - c'est ce que le Talmud insinue de toute évidence - pesait la malédiction. Les hostilités qui allaient s'ensuivre entre le fils de Timna et Israël, ne sont donc pas dues au hasard. Au contraire, elles résultent bel et bien de la décision des

\footnotetext{
${ }^{3}$ C. Westermann, Biblischer Kommentar, Genesis ; chap. 12-36, Neukirchen 1989, $2^{\mathrm{e}}$ édition, p. 686 ; S. D. Kunin, The Logic of Incest : A Structuralist Analysis of Hebrew Mythology, Sheffield, Academic Press, 1995, p. 201 commente ainsi les relations entre Amalec et Israël: "Those nations genealogically closest are ideologically more distant... genealogical closeness is inversely related to ideological closeness ». (« Ces nations les plus proches par la généalogie sont les plus éloignées par la culture... la proximité généalogique est inversement proportionnelle à la proximité culturelle »).

${ }^{4}$ Une version préliminaire de ce récit se trouve dans BerR 82, 14 (sur Gn 36, 12). Il n’y est pas question du souhait de Timna de se convertir au judaïsme, mais de son intérêt à faire partie, par le mariage, de la famille d'Abraham.

${ }^{5}$ Cf. Rachi sur Gn 36, 12.
} 
patriarches que le Talmud, eu égard aux épreuves endurées au cours des guerres successives contre les Amalécites, s'attache à considérer comme une erreur. Cette narration suggère que le refus essuyé par la princesse horite était inconsidéré. Au cours de son histoire, le peuple d'Israël allait payer cher l'exclusion de Timna.

Si notre interprétation du commentaire midrashique de l'épisode de Timna est correcte, elle se trouve en accord avec la plupart des représentations de la figure d'Amalec dans la littérature rabbinique. Dès que la figure d'Amalec y est abordée, un autre thème apparaît, thème que les rabbins interprètent de manière plutôt insolite. Nous nous attacherons dans ce qui suit à montrer que les exégèses des midrashim postbibliques se distinguent par le fait qu'elles s'occupent moins de l'ennemi et des détails de la lutte contre lui qu'elles ne s'attachent à poser la question de savoir ce qu'Israël a fait pour déclencher ces périlleux affrontements. Et les rabbins de chercher une réponse du côté des (mauvaises) décisions prises par le passé, que ce soit aux temps révolus des patriarches ou dans l'histoire plus récente. En partant de ces expériences, ils se penchent aussi sur ce qu'Israël devait faire ou éviter de faire pour se défendre contre Amalec ${ }^{6}$.

\section{L'interprétation de Ex 17, 8-16 dans l'exégèse historico-critique contemporaine}

Le premier récit biblique, et le plus connu, qui parle d'un combat entre Israël et Amalec ${ }^{7}$ débute par cette affirmation quelque peu abrupte : "Amalec vint combattre Israël » $(\mathrm{Ex} 17,8)$. Par la suite, le récit se détourne toutefois des actions sur le champ de bataille pour se concentrer sur les détails des mesures que prennent Moïse et ses collaborateurs pour contrer l'attaque. Le combat lui-même ne réapparaît qu'au verset 13.

8. Amalec vint (wayavo) combattre Israël à Rephidim.

9. Alors Moïse dit à Josué : Choisis-nous des hommes, sors, et combats Amalec ; demain je me tiendrai sur le sommet de la colline, la verge de Dieu dans ma main.

10. Josué fit ce que lui avait dit Moïse, pour combattre Amalec. Et Moïse, Aaron et Hur montèrent au sommet de la colline.

\footnotetext{
${ }^{6}$ Voir à ce sujet les exemples tirés du midrash Esther Rabba chez J. Neusner, Judaism and the Interpretation of Scripture. Introduction to the Rabbinic Midrash, Peabody, 2004, p. 119-121.

${ }^{7}$ Exode 17, 8-16.
} 
11. Lorsque Moïse élevait sa main, Israël était le plus fort ; et lorsqu'il baissait sa main, Amalec était le plus fort.

12. Les mains de Moïse étant fatiguées, ils prirent une pierre qu'ils placèrent sous lui, et il s'assit dessus. Aaron et Hur soutenaient ses mains, l'un d'un côté, l'autre de l'autre; et ses mains restèrent fermes jusqu'au coucher du soleil.

13. Et Josué vainquit Amalec et son peuple, au tranchant de l'épée.

14. L'Éternel dit à Moïse : Écris cela dans le livre, pour que le souvenir s'en conserve, et déclare à Josué que j'effacerai la mémoire d'Amalec de dessous les cieux.

15. Moïse bâtit un autel, et lui donna pour nom : l'Éternel ma bannière.

16. Il dit: Parce que la main a été levée sur le trône de l'Éternel, il y aura une guerre de 1'Éternel contre Amalec, de génération en génération. ${ }^{8}$

Dans une réflexion plus tardive sur ce récit (Dt 25, 19) que les biblistes considèrent comme un ajout postérieur à la source sacerdotale ${ }^{9}$, on peut lire que la mémoire des Amalécites sera " effacée », c'est-à-dire que ce peuple doit être complétement exterminé.

La recherche a longtemps considéré ces récits de l'affrontement entre Israël et Amalec au livre de l'Exode, comme dans d'autres passages bibliques, comme le reflet de souvenirs «d'anciens conflits à propos d'oasis et de pâturages avant la sédentarisation ${ }^{10}$. Les exégètes présumaient, pour étayer leur interprétation, que les Amalécites auraient été primitivement une tribu nomade vivant dans le désert du Néguev. En s'appuyant sur Juges 6, 3-5, 1 Samuel 15, 3 et 30, 17 on supposait ensuite que cette peuplade possédait des chameaux et qu'elle assurait sa subsistance en lançant des attaques armées (cf. également Jg 7, 12) ${ }^{11}$. De la première phrase du passage cité, Umberto Cassuto conclut que les Amalécites venaient d'assez loin :

Comme ils occupaient les abords au sud du pays de Canaan et qu'ils avaient forcément appris que les Israélites s'avançaient dans cette direction, ils voulurent éliminer le danger en envoyant un détachement de soldats chargés d'attaquer les

\footnotetext{
${ }^{8}$ Sauf indication contraire, la traduction française de la Bible hébraïque est celle de Louis Segond.

${ }^{9}$ W. Oswald, « Defeating Amalec. Defending the Constitution : The Political Theory of Ex 17, 8-16 », dans C. Berner et H. Samuel (éd.), The Reception of Biblical War Legislation in Narrative Contexts. Proceedings of the EABS Research Group "Law and Narrative” (Beihefte zur Zeitschrift für die alttestamentliche Wissenschaft 460), Berlin, 2015, p. 61-72 (ici : p. 70).

${ }^{10}$ A. Huwyler, s.v. «Amalek, Amalekiter», dans Calwer Bibellexikon, éd. par O. Betz, B. Ego et W. Grimm en collaboration avec W. Zwickel, Stuttgart, 2003, tome I, p. 72.

${ }^{11}$ Dans 1 Samuel 15, 5 il est toutefois question d'une ville peuplée par les Amalécites et d'un roi, Agag; ce qui s'accorde mal avec l'hypothèse d'un mode de vie nomade. Quant à Agag voir aussi 1 Sm 15, 8.9.20.32.
} 
Israélites dès le début de leur déplacement. Selon la tradition figurant dans un autre passage de la Torah [...] cette troupe commença par attaquer l'arrièregarde de la multitude des Israélites, ceux qui par faiblesse ou épuisement n'étaient pas en mesure de se défendre. ${ }^{12}$

On peut cependant opposer à cette interprétation historique le fait que les traces archéologiques et les témoignages - autres que ceux de la Bible - concernant Amalec nous font complétement défaut; et même en s'appuyant sur la Bible, cette prétendue tribu ne peut être localisée avec précision: le verset Nombre 14, 25 semble suggérer qu'Amalec et Canaan habitaient côte à côte dans la plaine tandis que $\mathrm{Nb} \mathrm{14,39-45}$ évoque plutôt un habitat en montagne. Aussi les recherches récentes se sont-elles détournées de toute spéculation sur l'histoire d'Amalec et des reconstructions d'événements historiques qui prétendent étayer ces récits, pour se concentrer sur l'analyse des textes vétérotestamentaires euxmêmes.

En règle générale, le point de départ de ces considérations est la place de la narration dans l'agencement littéraire du Pentateuque, la mise en contexte ( Sitz im Leben ») et la structure littéraire des textes. Pour Hans Christoph Schmitt, ce passage de l'Exode (17, 8-16) est un exemple qui illustre «que les traditions narratives telles qu'elles sont transmises par l'Ancien Testament n'ont pas principalement d'intérêt historiographique ${ }^{13}$. Partant de cette affirmation, le spécialiste de l'Ancien Testament et professeur à l'Université de Tübingen Wolfgang Oswald fait remarquer que les faits d'armes eux-mêmes restent plutôt à l'arrière-plan et que, par ailleurs, les contours de l'ennemi sont assez flous. Il s'agirait en premier lieu de Moïse, d'Aaron et de Hur, et de leur action défensive « sur le sommet de la colline » (Ex 17, 10b). Dans une perspective historique, ce texte pourrait ainsi traduire, sous forme de narration, une situation de faiblesse politique et militaire du peuple d'Israël pendant l'époque perse, donc celle des dernières années de l'exil

12 «Since they occupied approaches to the land of Canaan from the south, and undoubtedly they had heard that the Israelites were marching in that direction, they wished to forestall trouble by sending a detachment of soldiers to attack the Israelites at the commencement of their journey. According to the tradition reflected in another passage of the Torah [...] the first action taken by this troop was to attack the rearguard of the Israelite host, the weak and the weary who are unable to defend themselves ", U. Cassuto, A Commentary on the Book of Exodus, Jerusalem, 1987, p. 204.

${ }^{13}$ H.-C. Schmitt, «Die Geschichte vom Sieg über die Amalekiter Ex 17, 8-16 als theologische Lehrerzählung ", Zeitschrift für die alttestamentliche Wissenschaft 102, 1990, p. 335-344 (ici : p. 344). 
de Babylone ou peu de temps après celui-ci. La colline sur laquelle se tiennent Moïse et ses collaborateurs, devrait être considérée, estime-t-il, comme une allusion au Temple de Jérusalem, détruit par les Babyloniens, l'autel dressé sur la colline représenterait le culte provisoire de ceux qui reviennent de Babylone avant la reconstruction du Temple, le désert, le pays de Juda dévasté ; le peuple d'Israël serait « le corps politique de la Judée durant la première période perse» et Amalec «l'ennemi paradigmatique du moment, probablement Edom $»^{14}$.

Cette communauté fragile ne peut survivre à sa situation désespérée qu'à la seule condition de faire confiance à la Torah de Moïse ou d'accepter le prophète et successeur de Moïse [...] Tous ceux qui détenaient une charge, civile, militaire ou cultuelle, ont l'obligation d'agir en accord avec Moïse. Partant de là, plutôt que de fêter une victoire des temps reculés, l'épisode de l'Exode 17, 8 - 16 vise à consolider le système politique de son époque, à savoir la constitution telle qu'elle est définie dans le livre du Deutéronome. ${ }^{15}$

Il s'agirait en substance, selon cette interprétation, d'un texte conçu d'emblée comme allégorique par ses auteurs. Les développements qui suivent s'attacheront à montrer que l'interprétation qui prévaut dans le midrash rabbinique suit de manière analogue, dans des circonstances différentes, les schémas allégoriques et rend justice au texte biblique sous-jacent. Certes, les midrashim ne montrent aucun intérêt - après la seconde destruction du Temple par les Romains en 70 de l'ère chrétienne - à faire apparaître, à travers la colline mentionnée dans l'Exode, celle de Sion et la reprise espérée du culte sacrificiel; mais le message central quant à son orientation parénétique tend à coïncider avec l'intention mise en lumière par les recherches bibliques récentes. De même que les spécialistes actuels de l'Ancien Testament, les auteurs des midrashim ne conçoivent pas le texte biblique comme un récit historique, mais comme un appel, présenté sous forme de narration, à l'obéissance de ses lecteurs à la Torah. Même là où, dans quelques midrashim tardifs, un certain intérêt se manifeste de nouveau pour le contexte historique, la

\footnotetext{
${ }^{14}$ «The body politic of early Persian period Judah» et «The paradigmatic enemy of the time probably Edom », W. Oswald, «Defeating Amalec... », art. cit., p. 68.

${ }^{15}$ «This vulnerable community can survive under these dire straits if and only if they rely on the torah of Moses or if they accept the prophetic successor of Moses [...] All officeholders, be it a civic, a military, or a cultic office, are obliged to act in accordance with Moses. Advocating this idea, rather than celebrating a primeval victory, the episode Exod 17, 8-16 intends to strengthen the political system of its own time, and that is the constitution as it is defined in the book of Deuteronomy. ») W. Oswald, ibid.
} 
perspective essentiellement allégorique et en même temps parénétique relègue les aspects historiographiques à l'arrière-plan.

\section{Appel à la fidélité à la Torah : la mekhilta de Rabbi Yischma'el}

La mekhilta de Rabbi Yischma'el, le plus ancien commentaire rabbinique sur l'Exode, rédigé probablement vers la fin du $\mathrm{III}^{\mathrm{e}}$ siècle après J.-C., se situe dans cette perspective - non sans présenter une certaine ressemblance avec l'exégèse midrashique de l'épisode de Timna mentionnée plus haut - en pointant du doigt les manquements des Israélites. C'était pour avoir négligé la Torah que surgit le danger représenté par les Amalécites : plus précisément le manquement que le livre de l'Exode avait évoqué précédemment $(17,7)$ reprochant au peuple de s'en prendre à Dieu (« Le Seigneur est-il parmi nous ou non ?»). Le midrash commence dans le style d'une petihta (ouverture) classique, en mettant en parallèle le verset $($ Ex 17, 8) avec un verset du livre de Job, en apparence très éloigné, qui doit éclairer et expliquer le texte précité.

Rabbi Yehochu'a et rabbi Él'azar Hisma ${ }^{16}$ disent : ce verset (i.e. Ex 17, 8) reste obscur et s'éclaire à la lumière de Job $(8,11)$ : « Le jonc croît-il sans marais ? ». Le jonc peut-il donc pousser sans le marais et sans eau ? Ainsi Israël ne sera que s'il s'occupe des paroles de la Torah. Et c'est parce qu'ils se sont éloignés des paroles de la Torah que l'ennemi les a attaqués ; car l'ennemi ne vient qu'à cause du péché, à cause de la transgression. C'est pour cela qu'il est dit : « Amalec vint $»(\text { Ex 17, 8) })^{17}$.

De même que le jonc dépérit sans eau, estime le midrash, parce que la sécheresse entraîne la ruine, de même Israël ne peut prospérer sans la Torah parce que dans de telles circonstances «Amalec vient », pour ainsi dire par la force des choses. La suite de la mekhilta confirme cette orientation et met en lumière le péché d'Israël en associant à Israël (par un lien syntaxique quelque peu obscur) la formule «sans aucune crainte de Dieu » (welo yaré elohim) qui se trouve dans le passage parallèle du

\footnotetext{
${ }^{16} \mathrm{Il}$ s'agit apparemment de deux tannaïm de la $2^{\mathrm{e}}$ génération qui ont exercé leur activité d'environ 90 à 130 après J.-C. : Rabbi Yehochu'a ben Hananya, appelé simplement Rabbi Yehochu'a la plupart du temps, qui vivait à Peqi'in, et son élève Él'azar Hisma (cf. G. Stemberger, Einleitung in Talmud und Midrash, München, C.H. Beck, 1992, $8^{\mathrm{e}}$ éd., p. 78 sq. et p. 82). Tous deux s'occupaient de règles halakhiques en rapport avec l'agriculture.

${ }^{17}$ Voir la traduction de G. Stemberger, Mekhilta De Rabbi Jischma'el. Ein früher Midrasch zum Buch Exodus. Aus dem Hebräischen übersetzt und herausgegeben, Berlin, 2010, p. 218.
} 
Deutéronome $(25,18)$. Ce n'est pas l'ennemi perfide, comme le suggère une lecture littérale du texte ${ }^{18}$, mais le peuple d'Israël lui-même qui était «sans aucune crainte de Dieu» parce qu'il «n'avait pas d'actions méritoires à son actif $»^{19}$. C'est pour cela qu'il arriva à Rephidim - ce nom toponymique est interprété comme «faiblesse des mains » (rifeyon yadayim) - et fut talonné par Amalec : pour être puni ${ }^{20}$. La conséquence de cette interprétation est que le sens profond de la guerre sainte contre Amalec consiste à lutter contre ses propres péchés. Dans cette guerre, les paramètres de la victoire ne sont en rien dus aux qualités réelles, historiques, de l'ennemi, l'issue du combat dépend exclusivement de la persévérance avec laquelle Israël respecte la Torah et accomplit des actions méritoires.

\section{Les quatre empires ennemis et la culpabilité d'Israël : Exode Rabba}

Dans le midrash Exode Rabba (ExR), le commentaire sur Ex 17, 816 se trouve dans la seconde partie (plus tardive) de l'œuvre que la recherche distingue de ExR I en le désignant comme ExR II. Cette dernière partie contient des exégèses homilétiques se rapportant à Ex 1240 (dans les parashot 15-52) ${ }^{21}$, datant du début du $\mathrm{XI}^{\mathrm{e}}$ siècle environ ; on suppose toutefois qu'elle contient des traditions plus anciennes ${ }^{22}$. Comme nous le verrons, la visée parénétique des développements de ce texte consacré à Ex 17, 8-16 demeure d'abord cachée par des spéculations historico-métaphysiques. Pour expliquer l'expression « et Amalec vint» (wayavo 'Amaleq) cette composition midrashique anonyme se réfère une nouvelle fois à un verset très éloigné dans le canon biblique. Or ce verset - tiré, comme dans la mekhilta, du livre de Job - sera interprété dans la perspective de sa concordance avec Ex 17,

\footnotetext{
${ }^{18} \mathrm{C}$ 'est en effet ainsi qu'une compréhension grammatico-littérale du texte devrait reconstituer le texte et ainsi que toutes les traductions le comprennent.

${ }^{19}$ G. Stemberger, Mekhilta..., op. cit., p. 218.

${ }^{20} \mathrm{Ibid}$. Voir à ce sujet le Midrash Haggadol (MHG) commentant Dt 25, 17 : «sans crainte de Dieu - d'autres diront : C'est Israël qui n'a pas accompli d'actions méritoires qui les [sic] auraient sauvés » (S. Fisch, Midrash Haggadol on the Pentateuch. Deuteronomy, Jérusalem, Mossad Harav Kook, 1972, p. 576, 16-17).

${ }^{21} \mathrm{La}$ datation de ce midrash est rendue difficile par le fait qu'il renferme des mots empruntés au latin et au grec et qu'il utilise visiblement la littérature tannaïtique ; en revanche il ne comporte aucune citation du Talmud de Babylone. Les premiers auteurs qui citent ExR sont Arziel de Gérone ( $1^{\text {ère }}$ moitié du XIII ${ }^{\mathrm{e}}$ siècle) et Nahmanide (vers 1260).

${ }^{22}$ G. Stemberger, Einleitung..., op. cit., p. 304.
} 
8 ; un fait qu'on ne remarque qu'à la fin. On notera la forme verbale identique (wayavo - « il vint $»)$ :

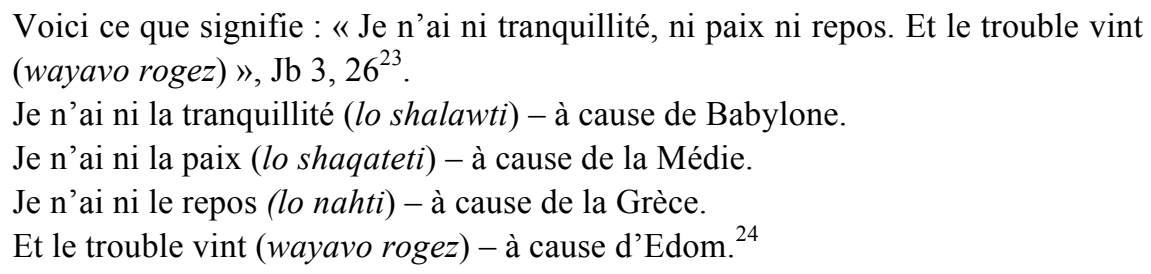

À première vue, ces mises en parallèle paraissent arbitraires. Méconnaissant en apparence les parallélismes de la poésie biblique, l'exégète interprète chaque partie du verset comme s'il se rapportait à une réalité historique différente. Ainsi voit-on évoquées quatre périodes historiques successives sans qu'il y ait dans le texte biblique le moindre indice matériel, surtout historique, chronologique ou territorial ${ }^{25}$. L'agencement du midrash présuppose évidemment le schéma sotériologique de Daniel 7, selon lequel l'histoire du peuple d'Israël peut être représentée comme une série d'affrontements avec quatre royaumes ennemis successifs. La rencontre avec ces quatre empires se reflète de façon concentrée dans le combat avec Amalec. En définitive, toute l'histoire du salut se retrouve dans ce seul verset de l'Exode $(17,8)$ interprété à la lumière de Job 3, 26. Dans cette mesure, on peut affirmer que Babylone et la Médie/la Perse aussi bien que la Grèce et Edom doivent être compris sous le signe d'Amalec. Cette attribution acquiert tout son sens dans la dernière partie du verset : Amalec dont le nom n'est toutefois pas mentionné à cet endroit, ni dans le paragraphe suivant du midrash, est, en sa qualité de petit-fils d'Ésaü, intimement lié à Edom, le « quatrième royaume ».

\footnotetext{
${ }^{23}$ On peut se demander si le rapprochement de l'extrait de Job avec Amalec n'est pas suggéré par l'identité du nom d'Éliphaz, le père d'Amalec $(\mathrm{Gn} 36,12)$ avec celui d'Éliphaz de Théman, l'ami de Job qui prend la parole à cet endroit.

${ }^{24}$ ExR XXVI, 1 (sur Ex 17, 8); cf. M. Hadas-Lebel, Jérusalem contre Rome, Paris, Cerf, 1990, p. 475 ; traduction d'A. Wünsche, Der Midrasch Schemot Rabba. Das ist die haggadische Auslegung des zweiten Buches Moses. Zum ersten Male ins Deutsche übertragen, Leipzig, 1882, (nouvelle édition bilingue - allemand et hébreu - éditée par Michael Krupp, Jérusalem, 2013), p. 198.

${ }^{25}$ Pour les stratégies de lecture du midrash, voir A. Samely, Forms of Rabbinic Literature and Thought. An Introduction, Oxford, University Press, 2007, 91-93. L'interprétation que nous proposons correspond aux $n^{\circ} 14,15$ et 22 du schéma de Samely.
} 
Or, il est essentiel de comprendre ici qu'Edom n'est autre que le cryptogramme qui désigne l'Empire romain. Cette identification se fait sur fond de récits bibliques qui relatent la participation des Edomites à la prise de Jérusalem par les Babyloniens en 587 avant J.-C. (Psaume 137, 7 ; Lamentations 4, 21 ; Abdias 10-14). Après la catastrophe de l'an 70 après J.-C. l'assimilation d'Edom aux démolisseurs du Temple est transférée sur les Romains ${ }^{26}$. Ainsi le «trouble» (rogez) évoqué dans ExR vise pour ses auteurs la puissance qui, à leur époque même, détermine les conditions de vie du peuple juif. Cependant l'identification d'Edom avec Rome n'a pas gommé l'association d'idée entre cette puissance et «Amalec »; elle l'a au contraire soulignée et, à travers l'interprétation allégorique, hissée à un autre niveau. Cela signifie : le récent désastre, la destruction du second Temple, n'était pas non plus dû au hasard; il peut être considéré comme étant programmé dans les « conditions génétiques » de la postérité d'Abraham. Par le biais des Romains, les destructeurs de Jérusalem et du Temple, le peuple d'Israël rencontre son ennemi de toujours, Amalec.

Des spéculations historiographiques qui retrouvent dans les textes bibliques antérieurs un écho des expériences actuelles ou contemporaines d'Israël (à l'époque des auteurs rabbiniques) avec des puissances ennemies, ne sont pas chose rare dans la littérature rabbinique ${ }^{27}$. Toutefois, dans le midrash Genèse Rabba, le rapprochement de certains versets avec les empires correspondants est étayé par des éléments exégétiques, tels que des (pseudo-)étymologies ou d'autres explications qui rendent intelligible le rapport établi avec les versets bibliques ${ }^{28}$. Mais

\footnotetext{
${ }^{26}$ Sur cette identification et l'image d'Edom dans le midrash palestinien Genèse Rabba (Bereshit Rabba) : voir M. Morgenstern, «The Image of Edom in Midrash Bereshit Rabbah », Revue de l'histoire des religions, 233, avril-juin 2016, p. 193-222 ; voir aussi : H. Inglebert, «Les images bibliques de Rome dans les textes juifs et chrétiens. Les Kittim, Babylone, Tyr et Ésaü-Edom », Revue de l'histoire des religions, 233, p. 223-254.

${ }^{27}$ Concernant les quatre royaumes successifs tels que le midrash les repère à maintes reprises dans les textes bibliques, voir M. Hadas-Lebel, Jérusalem contre Rome..., op. cit., p. 511-517. Pour ce qui est de l'arbitraire de la méthode du midrash, Hadas-Lebel se contente de cette formule lapidaire : dans le midrash « tout rythme quaternaire suffit donc à suggérer les quatre empires » (p. 476).

${ }^{28}$ Pour ce qui est des justifications midrashiques que l'on ne trouve pas dans ExR, voir par exemple BerR 16, 4 (sur Gn 2,14) : ce passage met en rapport les quatre fleuves du Paradis avec les quatre royaumes évoqués; chaque fleuve est interprété en fonction de sa forme linguistique, de sa sonorité : le Pishon correspond à Babylone par Habacuc 1, 8 : «Ses cavaliers volent (pashu) [comme l'aigle]»; le Guihon est identifié à la Médie grâce au rapprochement avec le mot gehon (ventre), employé en Gn 3, 14 et au serpent crachant son venin; le Tigre (
} 
ici, dans ExR, nulle explication, nul commentaire pour les trois premiers segments du midrash qui se réfèrent à Babylone, à la Médie et à la Grèce. Le seul indice matériel qui justifie la juxtaposition des deux versets Ex 17, 8a et la fin de la phrase de Job 3, 26 qui renvoient au royaume d'Edom... donc à l'Empire romain, c'est l'identité des deux formes verbales wayavo («et vint...»). Ainsi la construction du midrash met très nettement l'accent sur la fin du passage, le quatrième et dernier terme ; ceci correspond d'ailleurs à la structure formelle du texte de Job, où une affirmation (wayavo rogez, "le trouble vint») succède à une triple négation (lo shalawti lo shaqateti welo nahti), et les rassemble par association.

Dans la suite du midrash, une seconde construction en quatre parties apparait. Ce paragraphe débute par l'expression dé 'ah 'aheret (« une autre interprétation »). Toutefois, il ne s'agit pas au sens propre d'une exégèse distincte mais d'une interprétation qui s'appuyant sur Job 3, 26, une fois encore, présume donc l'exposé précédent et le prolonge.

En mettant en rapport les parties du verset de Job 3, 26 avec des expressions tirées du livre de l'Exode, la succession des quatre puissances étrangères mentionnées précédemment - Babylone, la Médie, la Grèce et Edom (Rome) - se trouve complétée par l'ennemi qui s'impose dans le contexte du livre de l'Exode: l'Égypte. À quoi correspond l'apparition des trois sauveurs Miriam, Aaron et Moïse, succession qui culmine évidemment dans le personnage de Moïse :

Je ne me suis pas remis du premier tourment que le Pharaon a décrété contre moi, comme il est dit : « Ils leur rendirent la vie amère (wa-yemarerou) » (Ex 1, 14). Et Dieu procura au peuple un sauveur, c'est-à-dire Miriam, dont le nom est dérivé de ha-maror ( «l'amertume »).

Je ne me suis pas reposé du deuxième tourment provoqué par l'ordre du Pharaon : «Si c'est un garçon, faites-le mourir » $(E x 1,16)$. Mais Dieu nous a envoyé un sauveur en la personne d'Aaron qui s'appelait ainsi à cause de la grossesse (ha-herayon).

Je ne me suis pas reposé après le troisième tourment que le Pharaon a causé : «Vous jetterez dans le fleuve tout garçon qui naîtra » (Ex 1, 22). Mais Dieu nous a envoyé un sauveur : Moïse (Mosheh) qui s'appelle ainsi à cause de l'eau ; car il est écrit : « Je l'ai retiré des eaux (meshitihou)» (Ex 2, 10).

Et des troubles se produisirent - c'est-à-dire Amalec. Car on lit ici « Amalec vint $\gg .29$

$(\underline{h} a d)$ et « irréfléchis » $(q o l)$ de la Grèce, tandis que l'Euphrate (Perat) renvoie à Edom, « devenu grand » (perah werabah) grâce à la bénédiction d'Isaac.

${ }^{29}$ ExR XXVI, 1 (sur Ex 17, 8) ; A. Wünsche, Der Midrasch..., op. cit., p. 198-199. 
Une nouvelle fois le point culminant de la construction qui, en guise de conclusion, revient au point de départ qu'est le verset de l'Exode $(17,8)$, n'est pas le troisième mais le quatrième et dernier terme. Le midrash souligne cette progression en faisant culminer l'interprétation - comme dans la mekhilta - sur des développements concernant l'obéissance à la loi divine. Cette transition commence par la question qui vise le contexte biblique immédiat du passage sur Amalec : «Qu'estil écrit avant? »(mah ketib lema 'alah $)^{30}$. On comprend par la suite que le danger qui émane d'Amalec était la conséquence du comportement précédent du peuple. Ce n'est pas pour rien que les Israélites en traversant le désert s'étaient plaints du manque d'eau et s'en étaient pris à Dieu. Le midrash interprète la question posée à Moïse, « Pourquoi nous as-tu fait monter hors d'Égypte ?»(Ex 17,3), comme une expression marquante de leur péché.

Et aussitôt Moïse se mit à prier, comme il est dit $($ Ex 17, 4) : «Moïse cria à l'Éternel de la manière suivante (lémor)». Que veut dire « de la manière suivante (lémor) »? En effet, il dit: Seigneur de l'univers! Révèle-moi s'ils veulent me tuer ou non. Et il reçut pour réponse : Que t'importe? « Passe devant le peuple ». ${ }^{31}$

Or, Rabbi Yehuda interprète la réponse de Dieu : « Passe devant le peuple» ('abor lifney ha- 'am) (Ex 17, 5a) dans le sens de l'ordre de « faire abstraction de leur péché » ('abor 'al hatayya' shelahem) ${ }^{32}$. Selon le midrash, Moïse qui, dans son différend avec les Israélites, craint pour sa vie, doit devenir semblable à Dieu et, comme le Créateur, rendre le bien pour le mal. C'est dans ce sens qu'il doit « faire abstraction de leur péché ». La verge de Moïse n'est pas une arme dans la lutte contre l'ennemi mais l'instrument du châtiment divin. Or, ce châtiment qui autrefois avait frappé les Égyptiens, devrait s'abattre sur les Israélites suite à leur péché. Cette verge avec laquelle Moïse avait jadis frappé le Nil, déclenchant les châtiments de Dieu, ne sera pas maintenant - chose surprenante - un moyen pour punir mais pour guérir.

Alors Dieu lui dit (à Moïse) : Je ne procède pas comme un être humain qui blesse avec un outil tranchant ${ }^{33}$ et guérit avec un cataplasme. Je guéris avec les

\footnotetext{
${ }^{30}$ ExR XXVI, 2 (sur Ex 17, 8) ; A. Wünsche, Der Midrasch..., op. cit., p. 199.

${ }^{31}$ Ibid.

${ }^{32}$ L'expression midrashique pourrait faire allusion à Ex 34, 7.

${ }^{33}$ Le mot hébreu 'izmel est sans doute dérivé du grec smilé (lancette, petit couteau). Voir A. Wünsche, Der Midrasch..., op. cit., p. 99 ; et S. Krauss, Griechische und
} 
moyens que j'ai employés en d'autres circonstances pour blesser. C'est pour cela qu'il est dit: «Et ta verge avec laquelle tu as frappé le fleuve, prends-la dans ta main » pour que tout le monde reconnaisse qu'elle est un instrument de la grâce divine. $^{34}$

Une nouvelle fois, ce n'est pas la lutte contre le peuple ennemi qui est le centre d'intérêt des rabbins, mais le rapport entre Moïse et le peuple. Dans la mesure où ce peuple est en proie au péché - et c'est précisément le cas au temps de l'exégète midrashique - il doit être exhorté à rester fidèle à la Torah par le biais de la narration. Malgré une certaine tendance historiosophique qui fait dans ce midrash - à la différence de la mekhilta - que les ennemis d'Israël ne s'effacent pas complétement derrière la parénèse, l'intention homilétique est nettement mise en avant ${ }^{35}$.

\section{La culpabilité d'Amalec (Edom) à l'égard de ses parents et grands-parents}

L'orientation «vers l'intérieur» des exégèses midrashiques précédentes menant à une réflexion sur l'obéissance du peuple à la Torah, devient plus manifeste encore si on la compare à une autre tradition exégétique qui s'est maintenue dans différents recueils midrashiques. Les réflexions de ce groupe de textes portent sur le fait que, selon la généalogie biblique, Amalec doit lui aussi être considéré comme un descendant d'Abraham. L'une des scènes du midrash Esther Rabba décrit une rencontre des Amalécites et du devin païen Balaam ( $\mathrm{Nb}$ 22), lequel, selon le Pentateuque, était chargé de maudire le peuple d'Israël pendant la traversée du désert. Interrogé par les Amalécites qui veulent savoir comment tenir tête aux Israélites, Balaam conseille :

Va et fais-leur la guerre ; car si tu leur fais la guerre, tu les vaincras. En effet, ils sont tributaires du mérite de leur ancêtre Abraham, et étant toi aussi un descendant d'Abraham (ben bno shel Avraham), tu participes à son mérite (bizekhouto shel Abraham). ${ }^{36}$

lateinische Lehnwörter in Talmud, Midrasch und Targum, Teil II, Hildesheim, Olms, 1964, p. 28.

${ }^{34}$ ExR XXVI, 2 (surt Ex 17, 8) ; A. Wünsche, Der Midrasch..., op. cit., p. 199.

${ }^{35}$ Pour le caractère de midrash homilétique d'Exode Rabba, voir G. Stemberger, Einleitung..., op. cit., p. 304.

${ }^{36}$ F. Gandus et M. Mergui (éd. et trad.), Le Midrash Rabba sur Esther, Paris, Gallimard, 2009 , p. 268 
À une époque où l'identification du personnage d'Edom avec l'Empire romain et donc avec le christianisme pouvait passer pour acquise dans l'esprit des rabbins, l'enracinement d'Amalec-Edom dans la lignée d'Abraham offre des possibilités particulières d'aborder les rapports extrêmement problématiques entre juifs et chrétiens.

\section{La durée de la vie d'Abraham abrégée}

Dans la Pesiqta de Rab Kahana, un midrash homilétique palestinien destiné à être lu lors des fêtes et certains shabbats - midrash qui date probablement $\mathrm{du} \mathrm{V}^{\mathrm{e}}$ siècle de notre ère ${ }^{37}$, - nous trouvons dans un passage en rapport avec Dt 25, 17 («Souviens-toi de ce que te fit Amalec...») des réflexions historiographiques sur Amalec qui prolongent la problématique de notre sujet. Une fois de plus, le midrash commence par mettre en relation le verset commenté avec un passage biblique en apparence fort éloigné, tiré ici des Psaumes. Mais contrairement aux textes précédents, il ne s'agit plus, dans les développements qui suivent, des péchés du peuple d'Israël mais de ceux d'Amalec ou d'Edom. Selon le midrash, le verset Dt 25, 17 se rapporte en effet à ce passage des Écritures :

Que l'iniquité de ses pères ('avotaw, i.e. les ancêtres d'Ésaü) reste en souvenir devant l'Éternel, et que le péché de sa mère ne soit point effacé » (Ps 109, 14). Les ancêtres d'Ésaü étaient-ils donc méchants? Au contraire, c'étaient des justes : Abraham, son grand-père (zeqeno), Isaac, son père (aviw). Et [pourtant] tu parles de « l'iniquité de ses pères » ('awon 'avotaw)!

Ce qui est visé, c'est [plutôt] le péché qu'il [Amalec-Edom] a commis contre ses ancêtres. Et de quelle manière a-t-il péché contre ses ancêtres? Tu trouveras [dans les Écritures] qu'Isaac a procédé de la force [virile] d'Abraham. Isaac est mort à 180 ans $(\mathrm{Gn} 35,28)$; Abraham a seulement atteint l'âge de 175 ans (Gn $25,7) !^{38}$

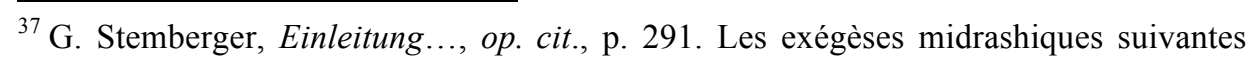
sont également transmises sous une forme similaire dans le Midrash Tanhouma, Ki-teze (Ed. Buber, Wilna, 1885, p. 34 sqq.) et dans le Midrash Haggadol (Ed. Fisch, op. cit., 571, p. 23 sqq). La Tanhouma est un midrash homilétique, lui aussi originaire de Palestine, que l'on date entre le $\mathrm{V}^{\mathrm{e}}$ et le $\mathrm{IX}^{\mathrm{e}}$ siècle (ibid., p. 300-301); le Midrash Haggadol, recueil d'exégèses probablement d'origine yéménite, est habituellement attribué à David ben Amram (XIII siècle). Voir ibid., p. 343-345.

${ }^{38}$ Pesiqta de Rab Kahana, Piska 3, 1; citée d'après: W. G. Braude et I. J. Kapstein (trad.), Pesikta de Rab Kahana. R. Kahana's Compilation of Discourses for Sabbaths and Festal Days, Philadelphia, 1975, p. 43.
} 
Ce fait rapporté par les Écritures se trouve confronté, dans le

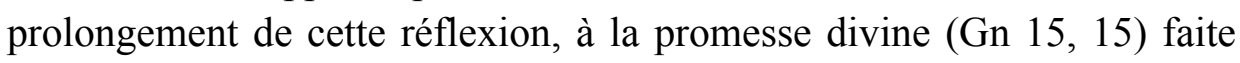
au patriarche de rejoindre ses ancêtres en paix à sa mort et d'être enterré à « un âge avancé » ${ }^{39}$. Selon cette interprétation, Dieu a fait entrevoir une longue vie au patriarche. De ce fait la question se pose de savoir pour quelle raison il n'a été accordé à Abraham qu'une vie amputée de cinq ans par rapport à celle de son fils.

Rabbi Yudan [a dit] au nom du Rabbi Aïbo, Rabbi Pinhas [a dit] au nom de Rabbi Levi ${ }^{40}$ : Au cours des cinq ans qui manquaient à la vie de notre aïeul Abraham [avant d'atteindre l'âge de 180 ans], Ésaü a commis deux graves infractions à la loi : il a eu des rapports sexuels avec une jeune fille fiancée, et il a tué un autre homme. De la première infraction, il est dit : «Et Ésaü revint des champs (hassadeh)» (Gn 25, 29). Or « champ » ne signifie rien d'autre qu'une fiancée, car il est dit : "Mais si c'est dans les champs (bassadeh) que cet homme rencontre la jeune femme fiancée, lui fait violence et couche avec elle » (Dt 22, 25]. [Quant au meurtre dont il s'est rendu coupable, on peut le déduire du fait qu'Ésaü] était "fatigué » ('ayef) (Gn 25, 29). "Fatigué » ne veut rien dire d'autre que «tuer», car il est écrit: "Malheureuse que je suis! Car je suis fatiguée ('ayefah); je succombe sous les meurtriers » $(\mathrm{Jr} 4,31){ }^{41}$

Comment concevoir les effets des méfaits, ici constatés, d'Ésaü sur la durée de vie d'Abraham ? Le patriarche est-il mort prématurément de chagrin en apprenant les péchés de son petit-fils ? S'agit-il d'un autre principe émanant de l'intervention divine dans l'histoire? Pourquoi les péchés d'Ésaü avaient-ils des conséquences pour son grand-père mais non pour son père? Faut-il supposer une sorte de détérioration de la qualité de la famille d'Abraham dans l'histoire du salut, par la faute d'Amalec-Ésaü ? Le midrash n'aborde aucune de ces questions. Au lieu

\footnotetext{
${ }^{39}$ Le texte midrashique parallèle (MHG 572, 5) reprend la forme araméenne du targoum Onkelos : sévou tava.

${ }^{40}$ Sur Rabbi Yudan et Rabbi Aïbo, deux amoräm palestiniens de la quatrième génération, dont l'activité s'étend sur le premier tiers du IV ${ }^{\mathrm{e}}$ siècle, voir G. Stemberger, Einleitung..., op. cit., p. 100. Rabbi Pinhas bar Chama, appartenant à la génération suivante, vécut également en Palestine. L'autorité à laquelle il se réfère, Rabbi Levi, désigne probablement l'amoraïm du même nom appartenant à la troisième génération (ibid., p. 96 et p. 102). Ces attributions - qu'il est de toute façon impossible de vérifier sont ici importantes dans la mesure où il s'agit chaque fois de transmetteurs palestiniens des traditions. En Palestine, la pression que représentait la christianisation progressive du pays pesait sensiblement sur les communautés juives.

${ }^{41}$ Pesikta de Rab Kahana. Piska 3, 1 (W. Braude et I. Kapstein, Pesikta..., op. cit., p. 43) Se référant à Abdias 5 ('im-gannabim ba'u-leka) on apprend ensuite par la bouche de Rabbi Zakkaï qu'Ésaü se serait également rendu coupable de vols (ibid., p. 43-44).
} 
de cela, il poursuit par une nouvelle énumération des infractions d'Ésaü (ou d'Amalec), classée par le nom des personnes qui en ont été également victimes.

\section{L'affaiblissement de la vue d'Isaac}

En lisant la liste de ces méfaits, on remarque que les faits de l'histoire biblique relatifs à la destinée des patriarches (et que la tradition ancienne, tel que le midrash palestinien Genèse Rabba [BerR], avait interprétée comme des circonstances concomitantes de l'action divine en vue du salut) sont présentés de façon négative et imputés à EdomAmalec. L'affaiblissement des yeux d'Isaac (Gn 27, 1) dont parle la Bible, est bien la condition sine qua non pour que Jacob puisse tromper son père et recevoir ainsi la bénédiction réservée à l'aîné. Plus ou moins en accord avec cette tendance narrative qui intègre dans une logique de salut les événements aboutissant à la méprise d'Isaac, déterminante pour la suite du récit, la vue affaiblie du patriarche se trouve rapprochée, dans Genèse Rabba de la «ligature d'Isaac » sur le bûcher dont fait état le chapitre 22 de la Genèse. Il reste à savoir si les yeux du patriarche s'affaiblirent d'effroi, s'il s'agissait d'un aveuglement lié à la mort imminente ou dû au fait qu'Isaac était proche des sphères célestes et que ses yeux ne pouvaient supporter les splendeurs de ce qu'ils avaient $\mathrm{vu}^{42}$. De ce fait, les jugements moraux s'effacent complétement dans l'interprétation que BerR donne de ces événements.

La Pesiqta de Rav Kahana, en revanche, impute la cécité du patriarche à Edom-Amalec. L'absence de toute intégration dans le récit qui le rendrait plausible (quel acte concret d'Ésaü a causé cette cécité ? Comment se représenter cette causalité ? Comment concilier l'idée de la culpabilité d'Ésaü, à l'origine de la cécité d'Isaac, avec le fait que celleci était de toute évidence nécessaire pour garantir la transmission de la bénédiction à son frère ?) ne gêne apparemment pas l'auteur.

\footnotetext{
${ }^{42}$ Voir BerR 65, 10 (sur Gn 27, 1) : selon ce texte, les anges du ciel se mirent à pleurer lorsqu'Abraham attacha son fils sur l'autel et leurs larmes tombèrent dans les yeux d'Isaac. Pour cette raison, ses yeux s'obscurcirent dans sa vieillesse. Selon une explication différente (ibid.) Isaac aperçut à ce moment-là la demeure de Dieu (shekhina) et fut ébloui au point que ses yeux refusèrent de fonctionner. Une autre interprétation explique l'affaiblissement de la vue du patriarche comme une mesure de protection divine: Isaac devait être préservé de voir les méfaits de son fils Ésaü (A. Wünsche, Der Midrasch..., op. cit., p. 311).
} 
Quel péché a-t-il commis contre son [propre] père ${ }^{43}$ ? Il a fait en sorte que ses yeux [ceux d'Isaac] s'affaiblirent [au point de ne plus rien voir] $(\mathrm{Gn} 27,1){ }^{44}$

\section{La blessure infligée à l'utérus de Rebecca}

Dans la suite du midrash, Rebecca, la mère d'Ésaü, se révèle être particulièrement touchée par les méfaits de son fils aîné.

Quel péché [Ésaü] a-t-il commis contre sa mère ${ }^{45}$ ? Rabbi Tanhoum bar Abun, Rabbi Yuda et Rabbi Néhémie ${ }^{46}$ et les rabbins [suivent des traditions différentes] : Rabbi Yuda dit : Lorsqu'il sortit du ventre de sa mère [Rebecca], il a sectionné son utérus (hatakh meterine shelah) pour qu'elle ne puisse plus enfanter (shelo teled $)^{47}$. C'est qu'il est dit : «Parce qu'il a poursuivi son frère avec l'épée [...], en étouffant sa compassion (weshihet rahamaw) » $\left(\mathrm{Am} \mathrm{1,11).}{ }^{48}\right.$

L'interprétation proposée par le midrash repose sur la polysémie de l'hébreu rehem, ici au pluriel: là où la lecture classique comprend « compassion» (sens admis du pluriel rahamim), le midrash infère le sens « utérus » (sens fréquent du singulier). Le midrash reprend ici une tradition provenant de Genèse Rabba qui présuppose et examine d'une manière particulière les rivalités et les tensions entre judaïsme et christianisme $^{49}$. Dans cette source, le thème de la matriarche sans enfant

\footnotetext{
${ }^{43}$ Dans ce qui suit, le midrash se réfère à l'expression « péché [contre] son père » du Psaume 109, 14a.

${ }^{44}$ Pesikta de Rab Kahana. Piska 3, 1 (W. Braude et I. Kapstein, Pesikta..., op. cit. p. 44).

${ }^{45}$ Le midrash se réfère à l'expression « péché [contre] sa mère » du Psaume 109, $14 \mathrm{~b}$.

${ }^{46}$ Il s'agit peut-être de Rabbi Yudan, mentionné par BerR 63,5 (cf. supra); Rabbi Néhémie, un tanna palestinien de la troisième génération (env. 130-160 après J.-C.), est également mentionné par BerR (sur Gn 25, 22). Rabbi Néhémie avait régulièrement des controverses avec Rabbi Juda bar Ilaï, si bien qu'il pourrait s'agir également de ce dernier. Manifestement, la mention des transmetteurs de traditions doit suggérer un lien entre la tradition ici présentée et le midrash Genèse Rabba. Quant à Rabbi Tanhoum bar Abba, un amora palestinien de la cinquième génération, voir G. Stemberger, Einleitung... op. cit., p. 102 et 298.

${ }^{47}$ Cf. le texte parallèle dans Tanhouma, ki teze, 4 (S. Buber, éd., Wilna, 1885, p. 35).

${ }^{48}$ Pesikta de Rab Kahana. Piska 3, 1 (W. Braude et I. Kapstein, Pesikta..., op. cit., p. 44). Selon Daniel Boyarin, l'utilisation de mots d'origine grecque dans les textes rabbiniques permet de conclure à des rapports intertextuels ou interculturels : "The use of the 'alien' word is not culturally innocent» ("L'usage de mots 'étrangers' n'est pas culturellement innocent »), D. Boyarin, Carnal Israel. Reading Sex in Talmudic Culture, Berkeley/Los Angeles/Londres, 1995, p. 43.

${ }^{49}$ Cf. M. Morgenstern, « Beobachtungen zur Neukonfiguration der Erzvätergeschichte im rabbinischen Midrasch: Die gebärmutterlose Geburt Rebekkas - oder: Warum Jakob Abraham errettete », Judaica. Beiträge zum Verstehen des Judentums 64, 2008,
} 
que rapporte la Genèse - selon Gn 25, 21, elle était «stérile » ('aqarah) - est amplifié par une narration: initialement Rebecca ne pouvait procréer n'ayant pas d'utérus (meterine lo hawah lah), nous dit ce midrash datant du $\mathrm{IV}^{\mathrm{e}}$ ou du $\mathrm{V}^{\mathrm{e}}$ siècle de notre ère, en ajoutant que Dieu en accomplissant un miracle lui en créa un (wegalaf lah haqadosh baroukh hou 'iqar meterine $)^{50}$ ! Ce texte précise que sa stérilité avait une cause anatomique! Mais Dieu lui en procura un, ne serait-ce que tardivement, qui la mit en mesure, grâce à ce miracle, de concevoir et de mettre au monde des enfants ${ }^{51}$. De même que notre midrash, Genèse Rabba, utilise l'emprunt au grec meterine ${ }^{52}$. Les auteurs du texte disposaient bien sûr de l'équivalent hébreu ou araméen rehem, mais leur intention était manifestement d'établir une association d'idées avec le monde grécophone. On peut imaginer une situation où des discussions (explicites ou implicites) et des polémiques les opposaient à des chrétiens qui se vantaient de la miraculeuse naissance de leur messie à Bethléem. Par la construction d'une naissance « encore plus miraculeuse », les juifs cherchaient ainsi à les surpasser : la mère de Jésus avait beau être vierge, la mère de Jacob (et donc d'Israël) ne disposait même pas d'un utérus au début!

Or, ce qui se présente dans ce midrash exégétique du $\mathrm{IV}^{\mathrm{e}}$ ou du $\mathrm{V}^{\mathrm{e}}$ siècle comme un miracle divin est l'objet, dans la tradition exégétique de la Pesiqta de-Rab Kahana, d'un acte sournois : la blessure infligée par Ésaü à sa mère. La présentation que l'on trouve ici traduit une réflexion sur le fait que la matriarche, selon le récit biblique, est non seulement la mère de Jacob, donc d'Israël, mais qu'elle a également mis au monde et ce, avant Jacob ! - le «méchant » (rasha) Ésaü. Après son expulsion du ventre maternel $(\mathrm{Gn} 25,25)$ l'impie aurait, à en croire le midrash, levé le poing contre son frère Jacob dans le but d'empêcher sa naissance et aurait par ce geste blessé l'utérus de sa mère. Le midrash commente ce comportement en citant le Psaume 58, 4 : «Les méchants sont pervertis dès le sein maternel (mérahem) ». Et cette précision se trouve justifiée

p. 37-53. Pour les allusions au christianisme, voir aussi EstR 7, 11 (A. Wünsche, Der Midrasch..., op. cit., p. 50). Il y est question de Haman, l'Agaguite, le fils d'un scélérat (ben rasha), qui finit par être condamné au supplice de la croix ('oleh lehatselav).

${ }^{50}$ BerR 63, 5 (sur Gn 25, 21); cf. A. Wünsche, Der Midrasch..., op. cit., p. 297 ; R. Lévy et J. Honigmann (trad.) Midrash Rabba Genèse, tome 2, Paris, Verdier, 2010, p. 51 : «Elle n'avait pas de matrice, et le Saint-béni-soit-Il en creusa une ».

${ }^{51}$ L'explication midrashique est d'une brièveté laconique. Aucune raison n'est donnée, mis à part le fait que le lecteur hébraïsant doit pouvoir déceler le radical ('qr) qui est à l'origine des mots « stérile » ('aqarah) et « au total» ('iqar).

${ }^{52}$ Cf. S. Krauss, Griechische..., op. cit., p. 334. 
par un verset au sens ambigu tiré du livre d'Amos $(1,11)$ : «Parce qu'il [Edom] a poursuivi son frère avec l'épée et qu'il s'est débarrassé de l'utérus »-c'est ainsi que le midrash, jouant sur les racines, comprend la formule weshihet rahamaw « en étouffant sa compassion ». De la sorte, estime Rabbi Néhémie, Ésaü serait responsable de l'impossibilité dans laquelle se trouvait Rebecca de donner la vie aux douze tribus alors qu'elle l'aurait mérité en raison de ses vertus et de sa piété ${ }^{53}$.

On ne peut déterminer si ce midrash vise, par le biais de ses développements sur Amalec, un ennemi réel de son époque. Mais à n'en pas douter, il exprime son indignation à l'égard d'un « Amalec » assimilé à « Ésaü », non pas en désignant et en fustigeant des actes concrets, mais en l'accusant en quelque sorte d'un délit sexuel à l'encontre de sa propre mère. Or cette mère est en même temps celle de Jacob, c'est-à-dire d'Israël ; on est donc en droit de penser que c'est la pire des choses qu'un auteur de midrashim puisse dire d'Amalec-Ésaü. Il s'agit en l'occurrence d'une hostilité qui a débuté avant même la naissance de Jacob-Israël. Elle est par conséquent indépendante de ce que ce frère jumeau, mais né après lui, ne pourra jamais être ou faire ; elle touche à toute la dimension ontologique des rapports entre Jacob et Ésaü. Il s'agit, dans ce contexte, d'une interprétation des rapports réciproques qui, sous l'angle de la responsabilité d'Israël, prend le contre-pied des textes mentionnés précédemment: si au départ il avait été question d'une sorte d'action punitive divine et qu'on affirmait alors que le peuple d'Israël pouvait détourner le malheur qui provenait de l'ennemi en obéissant à la Torah et en accomplissant de bonnes actions, les midrashim tardifs se focalisent

\footnotetext{
${ }^{53}$ Voir BerR 63, 6. Dans son commentaire sur Gn 26, 22, Rabbi Néhémie proposait d'attribuer à Rebecca elle-même la naissance des douze tribus d'Israël. Celle-ci, suggère-t-il, n'aurait pas mis au monde des jumeaux, mais douze enfants nés d'un même accouchement. On lit dans Gn 25, 22 : «Les enfants se heurtaient dans son sein ; et elle dit : S'il en est ainsi, pourquoi suis-je enceinte ?». "Rabbi Néhémie enseignait ceci : les douze tribus auraient dû émaner de Rebecca; c'est pour cela qu'il est écrit : l'Éternel lui dit: Deux nations sont dans ton ventre, cela fait deux; deux peuples se sépareront au sortir de tes entrailles, cela fait quatre ; l'un de ces peuples sera plus fort que l'autre, cela fait six ; le plus grand sera assujetti au plus petit, cela fait huit; les jours où elle devait accoucher s'accomplirent - et voici, il y avait deux jumeaux dans son ventre, cela fait dix ; le premier sortit entièrement roux, cela fait onze ; et ensuite sortit son frère, cela fait douze ». (GenR 63, 6 ; pour l'interprétation de ce texte voir M. Morgenstern, «Beobachtungen...», art. cité, 2008, p. 37-53). Il se peut que les différentes versions de cette narration traduisent un différend entre les rabbins quant à l'interprétation du fait que Rebecca était également la mère d'Ésaü (et donc d'Amalec) : Ésaü a-t-il par son seul statut de frère aîné empêché que la matriarche ne devînt directement la mère du peuple aux douze tribus ? Ou était-ce un acte de violence après sa naissance qui a contrecarré la faculté de Rebecca à enfanter?
} 
sur une hostilité envers Israël sur laquelle le peuple de Dieu n'a plus prise et qu'il ne peut détourner.

\section{Rebecca privée de funérailles publiques}

À cela s'ajoute, affirme le midrash, un dernier méfait qu'Ésaü aurait commis contre Rebecca. Il aurait empêché que sa mère bénéficiât de funérailles publiques à sa mort ${ }^{54}$. Dans un premier temps, la Pesikta de Rav Kahane s'étonne que le livre de la Genèse - contrairement à ce qui se passe pour les matriarches Sarah et Rachel $^{55}$ et aussi pour les patriarches Abraham, Isaac et Jacob ${ }^{56}$ - n'évoque pas le décès de Rebecca. Ce n'est qu'après coup que l'on apprend qu'elle fut enterrée « dans la caverne du champ de Macpéla, vis-à-vis de Mamré, dans le pays de Canaan ${ }^{57}$. Le midrash justifie cette particularité par le fait qu'après sa mort la question s'était posée de savoir qui pourrait l'accompagner vers sa dernière demeure. Étant donné qu'Abraham était déjà mort, qu'Isaac était privé de sa vue et que Jacob s'était enfui en Mésopotamie, il ne restait plus que « le méchant Ésaü ». Pour éviter que les gens ne maudissent «les seins qui ont allaité ce malfaiteur», on aurait organisé les funérailles pendant la nuit. Et Rabbi Yose ben Rabbi Hanina poursuit que pour étouffer l'affaire, la Bible (Gn 35, 8) raconterait à la place la mort et l'enterrement de la nourrice de Rebecca, Debora, sous le «chêne des pleurs » ('alon bakhot) à Bethel ${ }^{58}$. Par la suite, le midrash interprète le syntagme 'alon bakhot en le rapprochant $\mathrm{du}$ grec allos, comme l'indice «d'autres pleurs», à savoir des lamentations suite au décès de Rebecca ${ }^{59}$.

\footnotetext{
${ }^{54}$ Une version plus courte qui aborde ce thème se trouve déjà dans BerR 81, 5 (sur Gn 35,8 sq.). L'attention particulière portée dans ce midrash aux funérailles de Rebecca est d'autant plus frappante qu'il juge favorablement la présence d'Ésaü aux obsèques de son père Isaac $(\mathrm{Gn} 35,29)$ au chapitre suivant; selon BerR 82, 14 (sur Gn 36, 12) sa présence peut être considérée comme un acte respectant la Loi qui demande à honorer son père (kiboud 'av), et dans ce sens comme une «bonne action » (mitswah) - certes la seule qu'il ait accomplie! Toutefois, cet argument est avancé dans le contexte du souhait de Timna de rentrer dans la famille d'Abraham par le truchement d'Éliphaz.

${ }^{55}$ Concernant Sarah voir Gn 23, 1-20 ; pour Rachel Gn 35, 16-20.

${ }^{56}$ Voir Gn 25, 8 sq. (la mort d'Abraham) ; Gn 35, 28 sq. (la mort d'Isaac) et Gn 49, 33 (la mort de Jacob).

${ }^{57}$ Voir Gn 49, 31. On y apprend que sa sœur Léa est enterrée à côté d'elle.

${ }^{58}$ Pesikta de-Rab Kahana. Piska 3, 1 (W. Braude et I. Kapstein, Pesikta..., op. cit., p. 45).

${ }^{59}$ Ibid. ; voir à ce sujet S. Krauss, Griechische..., op. cit., p. 51.
} 
Il y a de bonnes raisons de penser que les considérations sur les obsèques de Rebecca, de la «mère d'Israël », constituent une réaction juive à différentes spéculations, au sein du christianisme, sur le lieu du décès de la mère de Jésus (ou de son assomption au ciel) qui se sont répandues au $\mathrm{V}^{\mathrm{e}}$ siècle en Palestine et qui ont apparemment donné lieu à toute sorte de discussions ${ }^{60}$. La proximité géographique de plusieurs églises byzantines consacrées à Marie et de la sépulture juive de Rachel entre Jérusalem et Bethléem atteste que les sanctuaires des deux religions ont pu très tôt entrer en concurrence ${ }^{61}$ - une constatation qui s'accorde avec l'hypothèse historique que les premières spéculations sur la Dormitio Mariae proviennent des milieux de juifs convertis au christianisme ${ }^{62}$. La tradition du «Palm of the Tree of Life » ${ }^{63}$ que l'on trouve sur des fragments syriaques de la fin du V $V^{\mathrm{e}}$ ou du début du VI $\mathrm{e}^{\mathrm{e}}$ siècle contient un récit qui nous intéresse dans ce contexte. Dans ce texte qui relate le dernier voyage de Marie, la rivalité entre juifs et chrétiens se manifeste d'une manière qui semble refléter les reproches que les rabbins adressent à Ésaü-Edom-Amalec. Voici le texte dans la paraphrase de Stephen Shoemaker :

Durant la nuit précédant sa mort [de Marie], Pierre adresse un long discours à ceux qui s'étaient rassemblés, et quand le jour se lève Marie se prépare à la mort. Les assistants sont miraculeusement plongés dans le sommeil, à la seule exception des apôtres et de trois vierges qui sont témoins de la venue du Christ entouré d'une légion d'anges. Puis le Christ accueille dans ses mains l'âme de Marie [...] Cependant, durant la procession funèbre, les dirigeants juifs ourdissent un complot pour détruire le corps de la Vierge. Mais lorsqu'ils tentent de mettre leur projet à exécution, ils sont tous frappés de cécité, sauf un seul homme, nommé Jephonias, qui se précipite sur le cercueil et tente de le renverser. Aussitôt que Jephonias s'empare du cercueil, un ange lui coupe les

\footnotetext{
${ }^{60}$ La première narration à ce sujet, le Liber Requiei Mariae (Livre de la Dormition de Marie) qui n'a survécu que dans une traduction éthiopienne, remonte peut-être à des traditions datant $\mathrm{du} \mathrm{IV}^{\mathrm{e}}$ siècle. La fête de l'Assomption remonte à une fête mariale instaurée par Cyrille d'Alexandrie au $\mathrm{V}^{\mathrm{e}}$ siècle. Voir S. J. Shoemaker, Ancient Traditions of the Virgin Mary's Dormition and Assumption, New York, Oxford University Press, 2004, p. 290-350.

${ }^{61}$ «Le rameau de l'arbre de vie». S. Shoemaker, Ancient Traditions..., op. cit., p. 8198, le montre en prenant l'exemple des traditions du kathisma et de deux sites au sud de Jérusalem qui peuvent être associés à cette tradition : une église près de Ramat Rachel, appelée « Old Kathisma » et l'église Mar Elias, nommée « New Kathisma Church ».

${ }^{61}$ Ibid., p. 212-214.

${ }^{62}$ Ibid., p. 212-214.

${ }^{63}$ Ibid., p. 32 sq.
} 
deux mains qui ne lui seront remises qu'au prix de sa conversion et de prières adressées à la Vierge $[\ldots] .{ }^{64}$

L'hypothèse d'une sorte de rapports polémiques réciproques entre la tradition mariale des chrétiens et celle de Rebecca chez les juifs concorde également avec des considérations sur l'absence de traditions relatives au décès de Marie. Il semblerait que ce silence eût été perçu comme une anomalie par le christianisme avant le V siècle. Alors que la date du décès de tous les autres saints était commémorée comme un jour de renaissance et d'admission au ciel (dies natalis), pour Marie on se contentait initialement de cérémonies célébrant sa maternité et la naissance de Jésus ${ }^{65}$. Plus tard, c'est dans le contexte d'une opposition très nette au judaïsme que se sont constituées des traditions sur la fin de sa vie sur terre. Les parallèles avec le récit midrashique des funérailles de la mère des jumeaux Jacob et Ésaü sont flagrants.

Si l'on fait abstraction du fait que les exégèses midrashiques citées s'attachent à interpréter différents textes bibliques ayant trait à Amalec, on constate, surtout dans les derniers points abordés ici, que toute référence concrète au petit-fills d'Ésaü a disparu. Le trait commun à tous ces textes, c'est que la réflexion sur Amalec s'efface complétement derrière celle sur Edom... à moins que ces deux motifs ne se confondent. En même temps, on a l'impression que le différend avec ce peuple ennemi prend une tournure particulièrement amère. Edom, auquel les juifs ne peuvent manifestement pas échapper dans le cadre des rivalités sotériologiques au sein de la famille d'Abraham, reste dans l'ombre d'Amalec, dont il a en quelque sorte pris les traits.

\footnotetext{
${ }^{64}$ " During the night before she [Mary] dies, Peter delivers a lengthy discourse to those who have gathered, and when morning arrives, Mary prepares herself for death. Those present are miraculously put to sleep, except only for the apostels and three virgins, who witness Christ's arrival with a company of angels. Christ then receives in his hands Mary's soul [...] During her funeral procession, however, the Jewish leaders plot to destroy the Virgin's body. Yet, when they attempt to carry out their plan, they are all stricken with blindness, with one exception, a man named Jephonias, who rushes her funeral bier and attempts to upset it. As soon as Jephonias grasps the bier, an angel cuts off his hands, and only by his conversion and prayers to the Virgin are they restored », S. Shoemaker, Ancient Traditions..., op. cit., p. 38. À ce sujet, voir aussi S. J. Shoemaker, "'Let Us Go and Burn Her Body' The Image of the Jews in the Early Dormition Tradition », Church History, 68, 1999, p. 775-823.

${ }^{65}$ S. Shoemaker, Ancient Traditions..., op. cit., p. 122.
} 


\section{Bibliographie}

Boyarin D., Carnal Israel. Reading Sex in Talmudic Culture, Berkeley/Los Angeles/Londres, 1995.

Braude W. G. et Kapstein I. J. (trad.), Pesikta de Rab Kahana. R. Kahana's Compilation of Discourses for Sabbaths and Festal Days, Philadelphia, 1975.

Fisch S. (trad.), Midrasch Haggadol on the Pentateuch. Deuteronomy, Jerusalem, Mossad Harav Kook, 1972.

S. D. Kunin, The Logic of Incest : A Structuralist Analysis of Hebrew Mythology, Sheffield, Academic Press, 1995.

Gandus F. et Mergui M. (éd. et trad.), Le Midrash Rabba sur Esther, Paris, Gallimard, 2009.

Hadas-Lebel M., Jérusalem contre Rome, Paris, Cerf, 1990.

Hieke T., Die Genealogien der Genesis (HBS 39), Fribourg-en-Br., 2003.

Huwyler A., s.v. «Amalek, Amalekiter», dans Calwer Bibellexikon, éd. par O. Betz, B. Ego et W. Grimm en collaboration avec W. Zwickel (éd.), Stuttgart, 2003, tome I, p. 72.

Inglebert H., "Les images bibliques de Rome dans les textes juifs et chrétiens. Les Kittim, Babylone, Tyr et Ésaü-Edom», Revue de l'histoire des religions, 233, 2016, p. 223-254.

Krauss S., Griechische und lateinische Lehnwörter in Talmud, Midrasch und Targum, Teil II, Hildesheim, Olms, 1964.

Lévy R. et Honigmann J. (trad.) Midrash Rabba Genèse, Paris, Verdier, 2010.

Morgenstern M., «Beobachtungen zur Neukonfiguration der Erzvätergeschichte im rabbinischen Midrasch: Die gebärmutterlose Geburt Rebekkas - oder: Warum Jakob Abraham errettete », Judaica. Beiträge zum Verstehen des Judentums, 64, 2008, p. 37-53.

Morgenstern M., "The Image of Edom in Midrash Bereshit Rabbah », Revue de l'histoire des religions, 233, 2016, p. 193-222.

Neusner J., Judaism and the Interpretation of Scripture. Introduction to the Rabbinic Midrash, Peabody, 2004.

Oswald W., «Defeating Amalec. Defending the Constitution: The Political Theory of Ex 17, 8-16», dans C. Berner et H. Samuel (éd.), 
The Reception of Biblical War Legislation in Narrative Contexts. Proceedings of the EABS Research Group "Law and Narrative", (BZAW, 460), Berlin, 2015, p. 61-72.

Samely A., Forms of Rabbinic Literature and Tought. An Introduction, Oxford, University Press, 2007.

Schmitt H.-C., « Die Geschichte vom Sieg über die Amalekiter Ex 17, 816 als theologische Lehrerzählung », ZAW, 102, 1990, p. 335-344.

Shoemaker S. J., "'Let Us Go and Burn Her Body' The Image of the Jews in the Early Dormition Tradition », Church History, 68, 1999, p. $775-823$.

Shoemaker S. J., Ancient Traditions of the Virgin Mary's Dormition and Assumption, New York, Oxford University Press, 2004.

Stemberger G., Einleitung in Talmud und Midrash, München, C.H. Beck, 1992, 8 éd.

Stemberger G. (trad.), Mekhilta De Rabbi Jischma'el. Ein früher Midrasch zum Buch Exodus. Aus dem Hebräischen übersetzt und herausgegeben, Berlin, 2010.

Westermann C., Biblischer Kommentar, Genesis; chap. 12-36, Neukirchen, 1989, $2^{\mathrm{e}}$ éd.

Wünsche A. (éd. et trad.), Der Midrasch Schemot Rabba. Das ist die haggadische Auslegung des zweiten Buches Moses. Zum ersten Male ins Deutsche übertragen, Leipzig, 1882, (nouvelle édition bilingue éditée par M. Krupp, Jérusalem, 2013). 